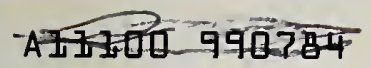

NBS

NBSIR 81-1644

A】llOb ๆ79215

PUBLICATIONS

\title{
AN APPROXIMATE EXPRESSION FOR THE PRINCIPAL BEAMWIDTHS OF DIRECTIVE ANTENNAS IN TERMS OF APERTURE FIELDS
}

Arthur D. Yaghjian

Electromagnetic Fields Division National Engineering Laboratory National Bureau of Standards Boulder, Colorado 80303

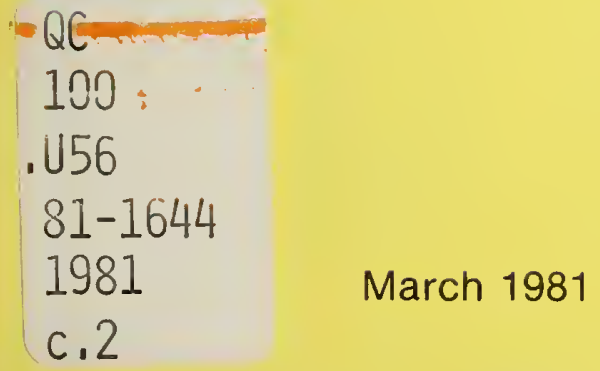

c.2 



\section{AN APPROXIMATE EXPRESSION FOR THE PRINCIPAL BEAMWIDTHS OF DIRECTIVE ANTENNAS IN TERMS OF APERTURE FIELDS}

Arthur D. Yaghjian

Electromagnetic Fields Division National Engineering Laboratory National Bureau of Standards

Boulder, Colorado 80303

March 1981

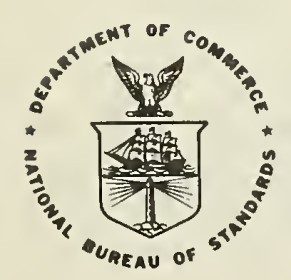

U.S. DEPARTMENT OF COMMERCE, Malcolm Baldrige, Secretary 

An Approximate Expression for the Principal Beamwidths of Directive Antennas in Terms of Aperture Fields

Arthur D. Yaghjian

An approximate, but general, formula for the half-power prinicpal beamwidths of directive antennas is derived from the Fourier transform of the aperture electric field. The derivation, which can be accomplished by expanding the transform in a Taylor series, is greatly streamlined by introducing a Gaussian beam approximation. The formula, which also relates beamwidth to directivity indirectly through simple integrations of the aperture field, reduces to a very compact expression in terms of the zero and second order moments of the aperture distribution for linearly polarized, uniformly phased aperture fields.

Key words: Antenna beamwidths; aperture fields; directive antennas.

Because the far field of an arbitrary antenna can be expressed exactly in terms of a double Fourier transform of the transverse electric field on an "aperture" plane in front of the antenna [1], the directive gain function of an antenna can also be expressed simply in terms of the aperture transverse electric field $\bar{E}_{a}$ by the following formula:

$$
D(\theta, \phi)=\frac{2 \pi Y_{0}}{\lambda^{2} P_{0}} \cos ^{2} \theta\left|\left(1-\sec \theta \hat{e}_{z} \hat{e}_{r} \cdot\right) \int_{A} \bar{E}_{a} \exp \left(-i \hat{e}_{r} \cdot \bar{R}_{a}\right) d A\right|^{2} .
$$

We will be concentrating on the main beam of directive antennas which are electrically large (in both aperture dimensions) with fairly uniform 
phase $^{\dagger}$ for each significant polarization in the transverse aperture field, thus producing a narrow main beam. (The term "directive" will be used throughout to denote specifically this class of antennas.) In the main beam of such antennas $\theta^{2}$ is much less than 1 radian ${ }^{2}$, and (1a) is well approximated by the simplified formula, ${ }^{\dagger \dagger}$

$$
D(\theta, \phi)=\frac{2 \pi Y_{0}}{\lambda^{2} P_{0}}\left|\int_{A} E_{a} \exp \left(-i k \hat{e}_{r} \cdot \tilde{R}_{a}\right) d A\right|^{2} .
$$

The coordinates in (1) are defined in figure $1, k=2 \pi / \lambda$ is the freespace propagation constant, $\lambda$ is the wavelength, $Y_{0}$ is the wave admittance, $A$ is the designated planar aperture which is truncated to a finite size, and $\exp (-i \omega t)$ time dependence for the fields has been suppressed. If the power $P_{0}$ radiated by the antenna is approximated by integrating $.5 Y_{0}\left|\vec{E}_{a}\right|^{2}$ over the aperture plane, (1) reduces, for its maximum value, to the familiar formula for directivity $D_{0}$ in terms of aperture fields $([1 \mathrm{a}]$, eq $(3.37))$,

$$
D_{0}=\frac{4 \pi}{\lambda^{2}} \frac{\left|\int_{A} \bar{E}_{a} d A\right|^{2}}{\int_{A}\left|E_{a}\right|^{2} d A} \text {. }
$$

The direction of maximum gain has been chosen to lie along the $z$-axis $(\theta=0)$, i.e., $D_{\theta}(0) \equiv \frac{\partial D}{\partial \theta}(0, \phi)=0$.

The main purpose of this communication is to derive a simple approximate formula, similar to (2) for half-power ( $3 \mathrm{~dB}$ ) beamwidths of directive antennas in terms of aperture fields. We can accomplish this by expanding (1) in a Taylor series about $\theta=0$, thereby generating a generatized moment expansion, and discarding terms which are negligible for directive antennas. However, it turns out that the same basic

This restriction can be relaxed for many aperture illuminations (e.g., see (16)), and the central result (10) remains a good approximation.

t†Equation (1b) is a decent approximation to (1a) for fairly large $\theta$; e.g., when $\theta=15$ degrees, $D(\theta, \phi)$ determined from (1a) and (1b) differ by only a few percent. 
approximate formula (10) which results for principal beamwidths can be derived in a much less complicated way by assuming the directive gain within $-3 \mathrm{~dB}$ of the main-beam axis obeys a Gaussian curve with respect to $\theta$; specifically,

$$
D(\theta, \phi)=D_{0} e^{-g(\phi) \theta^{2}}
$$

(Far-field patterns determined from measurements indicate that ( 3 ) is satisfied down to $-3 \mathrm{~dB}$ to a high degree of accuracy for directive antennas.)

Observe in (3) that the beam is not assumed to be circularly symmetric but can vary azimuthally with $\phi$. However, the azimuthal function $g(\phi)$ is restricted by the fact that for finite-sized apertures (1) demands all derivatives of $D$ with respect to $x$ and $y$ be continuous. Since $x=r \sin \theta \cos \phi$ and $y=r \sin \theta \sin \phi$, a straightforward application of the chain rule to any function $D(\theta, \phi)$ with continuous second derivatives proves that

$$
D_{\theta \theta}(0, \phi)=D_{x x}^{0} \cos ^{2} \phi+D_{x y}^{0} \sin 2 \phi+D_{y y}^{0} \sin ^{2} \phi,
$$

where subscripts indicate their respective partial derivatives, and the superscript 0 denotes the values of the partial derivatives $D_{x x}, D_{x y}$, and $D_{y y}$ at $(x, y, z)=(0,0,1), D_{x x}^{0}, D_{x y}^{0}$, and $D_{y y}^{0}$ are constants for chosen $x y$ axes; however, $-D_{\theta \theta}(0, \phi)$, the curvature of $D(\theta, \phi)$ at $\theta=0$, varies, in general, as a function of $\phi$.

For the sake of some simplification, we can choose the $x y$ axes along principal directions defined so that $D_{x y}^{0}=0$. (A straightforward rotation of coordinates proves such a choice is always possible [2]. For many linearly polarized antennas, these principal directions will coincide with "E-plane" and "H-plane" cuts.) Noting from (3) that

$$
D_{\theta \theta}(0, \phi)=-2 D_{0} g(\phi),
$$

we can then express $g(\phi)$ more specifically in terms of curvature as 


$$
g(\phi)=\frac{-1}{2 D_{0}}\left[D_{x x}^{0} \cos ^{2} \phi+D_{y y}^{0} \sin ^{2} \phi\right]
$$

A formal expression for the half-power beamwidth $\theta_{B}$, which is a function of $\phi$, follows directly from (3);

$$
\theta_{B}=2 \sqrt{\ln 2} \sqrt{\frac{1}{g(\phi)}},
$$

with, of course, $g(\phi)$ given by (6). By inspection of (4) or (6) we obtain the elementary theorem of differential geometry [2], that the principal curvatures along the principal directions $(\phi=0$ or $\pi)$ and $\left(\phi=\frac{\pi}{2}\right.$ or $\left.\frac{3 \pi}{2}\right)$ are the minimum and maximum curvatures (or vice versa). Hence from (7) and (6) the "principal beamwidths" along the principal directions are the minimum and maximum beamwidths given by

$$
\theta_{B}^{X}=2 \sqrt{2 \ln 2} \sqrt{\frac{-D_{0}}{D_{X X}^{0}}},
$$

$$
\theta_{B}^{y}=2 \sqrt{2 \ell n 2} \sqrt{\frac{-D_{0}}{D_{y y}^{0}}} .
$$

Moveover, $\theta_{B}$ from (7) can be rewritten for all $\phi$ in terms of the principal beamwidths from (8);

$$
\theta_{B}(\phi)=\frac{\theta_{B}^{x} \theta_{B}^{y}}{\sqrt{\left(\theta_{B}^{x}\right)^{2} \sin ^{2} \phi+\left(\theta_{B}^{y}\right)^{2} \cos ^{2} \phi}} .
$$

Equation (9) is the representation of an ellipse in polar coordinates, and thus expresses explicitly that the $-3 \mathrm{~dB}$ contour for narrow main beams approximates an ellipse.

All that remains to finding the beamwidth in terms of aperture fields is to evaluate the partial derivatives $D_{x x}^{0}$ and $D_{y y}^{0}$ from (1). This straightforward exercise in calculus finally yields for directive 
antennas,

$$
\left[\begin{array}{l}
x \\
B \\
y \\
B
\end{array}\right]=2 \sqrt{\ln 2} \frac{\left|\int_{A} \bar{E}_{a} d A\right|}{k \sqrt{\operatorname{Re}\left[\int_{A} \bar{E}_{a}\left[\begin{array}{l}
x_{a}^{2} \\
y^{2} \\
a
\end{array}\right] d A \cdot \int_{A} \bar{E}_{a}^{*} d A\right]-\left|\int_{A} \bar{E}_{a}\left[\begin{array}{l}
x \\
y \\
y
\end{array}\right] d A\right|^{2}}},
$$

For most directive antennas used in practice, $E_{a}$ exhibits a reasonable degree of even symmetry with respect to $x$ and $y$ axes whose origin is located at a central point in the aperture. For these antennas the second term in the denominator of (10) is negligible because $x_{a}$ and $y_{a}$ are odd functions with respect to the origin. For a perfectly symmetric (even with respect to $x$ and $y$ ) aperture field, or for a linearly polarized, uniformly phased aperture field, the origin can always be chosen to make this second term exactly zero, i.e.

$$
\int_{A} E_{a}\left|\begin{array}{l}
x \\
y \\
a
\end{array}\right| d A=0
$$

Recall also that the orientations of the $x y$ axes have been chosen along principal directions defined such that $D_{x y}^{0}=0$, which for the above stated aperture fields satisfying (11a), transiates from (1b) to

$$
\int_{A} \bar{E}_{a} x_{a} y_{a} d A=0
$$

If the transverse electric field is given or measured, an estimate of the beamwidths in (8) and (9) is provided through evaluation of the four (and usualiy three) independent aperture area integrations in (10). For linearly polarized, uniformly phased aperture fields, (10) and (11) reduce to a more compact form which allows $\theta_{B}^{x}$ and $\theta_{B}^{y}$ from (8) to be written in convenient "moment" terminology,

$$
\theta_{B}^{x}=\frac{2 \sqrt{\ln 2}}{k} \sqrt{\frac{\mu_{00}}{\mu_{20}}}
$$




$$
\begin{aligned}
& \theta_{B}^{y}=\frac{2 \sqrt{\frac{2 n}{2}}}{k} \sqrt{\frac{\mu_{00}}{\mu_{02}}}, \\
& \left(\mu_{10}=\mu_{01}=\mu_{11}=0\right)
\end{aligned}
$$

where $\mu_{m n}$ is the joint moment defined by

$$
u_{m n}=\int_{A}\left|\vec{E}_{a}\right| x^{m} y^{n} d x d y \text {. }
$$

For a circularly symmetric beam, $\theta_{B}^{x}=\theta_{B}^{y}=\theta_{B}^{c}$ and (12) combine with (9) to produce

$$
\theta_{B}^{C}=\frac{2 \sqrt{2 \ell n 2}}{k} \sqrt{\frac{\int_{A}\left|E_{a}\right| d A}{\int_{A}\left|E_{a}\right| R_{a}^{2} d A}} .
$$

Equations (10), (12), and (14) are approximate expressions for the beamwidth which approach in simplicity of form and ease of evaluation the corresponding approximate expression (2) for directivity. Although (10), (12), and (14) were derived by assuming a Gaussian main beam pattern down to $-3 \mathrm{~dB}$, it is emphasized that this is an unnecessary artifact introduced to greatly streamline their derivation. The same equations except for a factor $\sqrt{2-\sqrt{2}}=0.77$ replacing $\sqrt{\ell n 2}=0.83$ emerge directly from (1) by expanding $\sqrt{D(\theta, \phi)}$ in a Taylor series about $\theta=0$, retaining terms out to the fourth power in $\theta$, setting $D_{\theta}(0)=0$, and showing that the third and fourth terms are negligible for directive antennas. If the $\sqrt{\ell n 2}$ factor in (12) is replaced by $\sqrt{2-\sqrt{2}}$, (12) becomes identical to Spencer's result for two-dimensional scalar patterns from uniform-phase, symmetric aperture distributions ([3], Sec. 6.6).

Table I compares beamwidths computed from the approximate expression (14) with the exact values obtained by Hansen [4] and Silver ([3], Table 6.2) for circular aperture distributions with different amplitude tapers. The maximum discrepancy of 3 percent between exact and approximate values occurs for the uniform distribution (no taper). 
Finally, we estimate $\theta_{B}^{C}$ for the same circular aperture distributions using the common, rule-of-thumb relationship [5] which emerges between directivity and principal beamwidths when the total radiated power is roughly approximated by the power in the "equivalent" Gaussian beam (3). Specifically, insert $D(\theta, \phi)$ from (3) into the identity,

$$
1=\frac{1}{4 \pi} \int_{0}^{2 \pi} \int_{0}^{\pi} D(\theta, \phi) \sin \theta d \theta d \phi \text {, }
$$

perform the integration for large $g(\phi)$ (narrow beams), and obtain after substitution from (8)

$$
D_{0}=\frac{16 \text { ln } 2}{\theta_{B}^{x} \theta_{B}^{y}}=\frac{11.09}{\theta_{B}^{x} \theta_{B}^{y}} \text {. }
$$

This relationship, which has been derived by Ko [6] for circular beams $\left(\theta_{B}^{X}=\theta_{B}^{y}\right)$, represents somewhat of a compromise between Kraus's formula [5], $D_{0}=4 \pi / \theta_{B}^{X} \theta_{B}^{y}$, and Tai and Pereira's formula [7], $D_{0}=16 \ln 2 / 1 / 2\left(\theta_{B}^{x^{2}}+\theta_{B}^{y^{2}}\right)$.

The values of $\theta_{B}^{C}$ calculated from (15) (with $D_{0}$ evaluated from (2)) are included in table $I$. On the average they do not lie as close to the exact beamwidths as the values calculated from (14). But this would be expected since the derivation of (15), unlike (14), depends strongly upon the crude approximation that the total radiated power is contained in an equivalent Gaussian beam. For antennas with pattern shape factors or beam efficiencies differing appreciably from unity [5b], the relationship (15) between directivity and principal beamwidths must be modified by these parameters to remain useful, whereas $(10)$ continues to yield a reliable estimate of beamwidths because it inherently accounts for variations in beam efficiencies and pattern shape factors. This is a distinct advantage of (10) because often pattern shape factors and beam efficiencies are not readily available.

As a simple yet graphic example of an antenna aperture distribution 
for which (15) fails but (10) gives an accurate approximation to the beamwidths, consider the electrically-large square aperture of side $S$ illuminated with a linearly y-polarized transverse electric field

$$
E_{y}=1+\beta e^{i k x \sin \alpha}, \quad\left(0<\beta<1, \frac{2 \lambda}{S}<\alpha<\frac{\pi}{2}\right) .
$$

Such an antenna would have its mainbeam near $\theta=0$ and a grating lobe near $\theta=\alpha$. Its mainbeam is nearly circular with beamwidth and directivity given approximately by $.89 \lambda / S$ and $4 \pi S^{2} / \lambda^{2}\left(1+\beta^{2} \cos \alpha\right)$, respectively. Thus, the beamwidth and directivity of this antenna greatly deviate from the relationship (15) for non-negligible $\beta^{2} \cos \alpha$. However, application of the formula (10) yields approximate beamwidths,

$$
\theta_{B}^{X} \approx \theta_{B}^{y} \approx .92 \lambda / S,
$$

which are within a few percent of the actual beamwidths. 


\section{TABLE 1}

Exact and approximate values of beamwidth for circular aperture distributions $\left\{b+\left[1-\left(R_{a} / a\right)^{2}\right]^{n}\right\}$. ( $a$ is the radius of the aperture.)

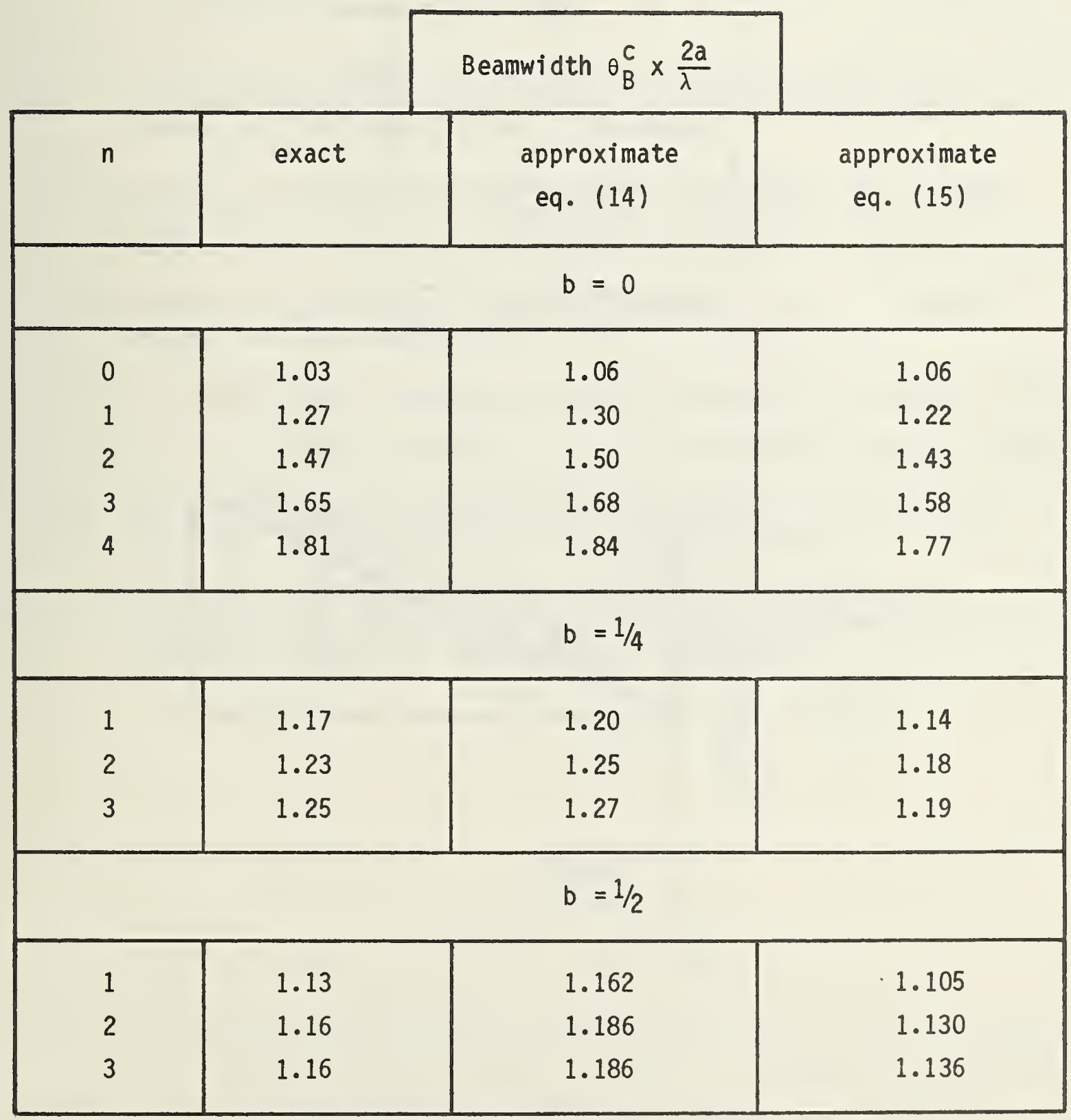




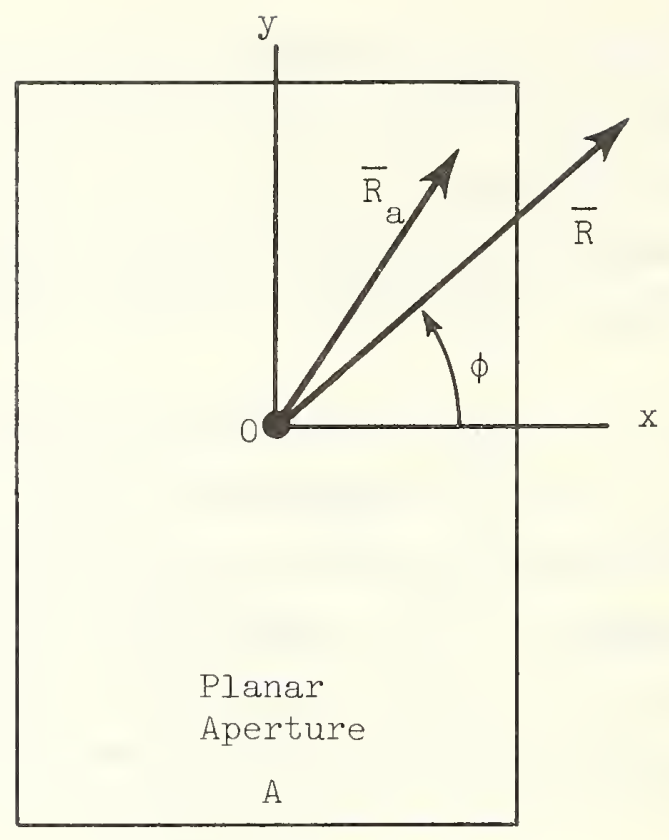

Front View

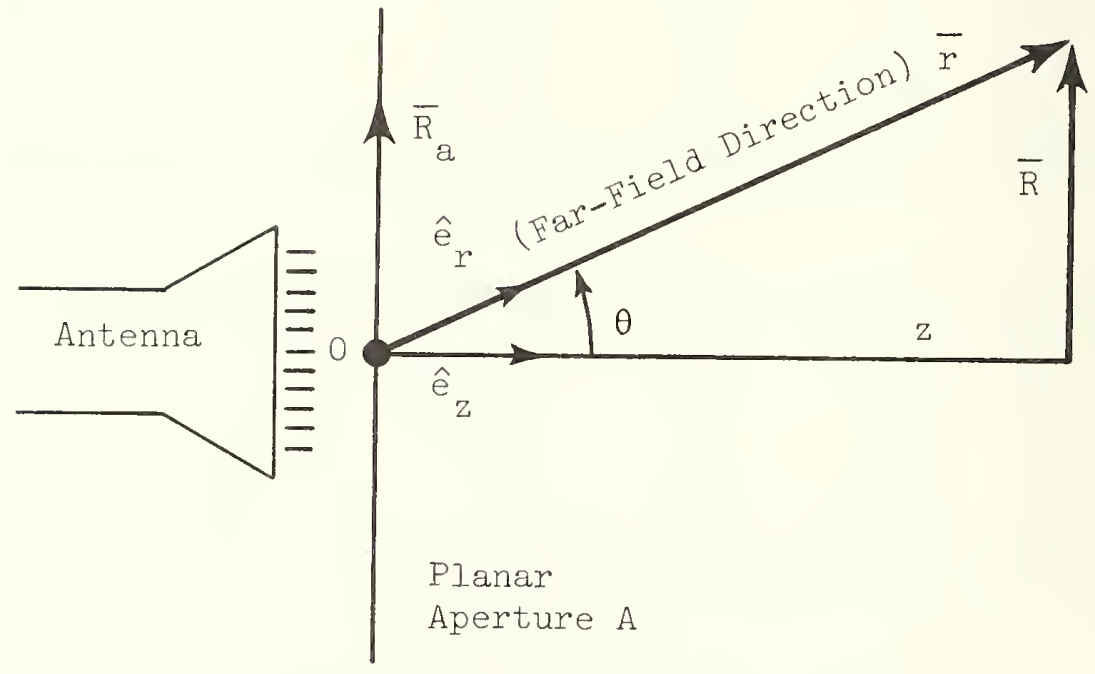

Side View

Figure 1. Definition of coordinates for far-field and aperture integration;

$$
\bar{R}_{a}=x_{a} \hat{e}_{x}+y_{a} \hat{e}_{y} \text {. }
$$




\section{REFERENCES}

[1] a) Collin, R.E., and Zucker, F.J., Antenna Theory, part I, ch. 3. McGraw-Hi11, New York (1969).

b) Kerns, D.M., Plane-wave scattering-matrix theory of antennas and antenna-antenna interactions: formulation and applications, J. of Res. Nat. Bur. of Stand., vol. 80B, pp. 5-51 (Jan. -Mar. 1976).

[2] Gauss, K.F., General Investigation of Curved Surfaces, ch. 1, sec. 8, Raven Press, New York (1965) (translated from the Latin and German by A. Hiltebeitel and J. Morehead).

[3] Silver, S., Microwave Antenna Theory and Design, ch. 6, Dover Publications, New York (1965) (first published by McGraw-Hill in 1949).

[4] Hansen, R.C., Microwave Scanning Antennas, I, ch. 1, Academic Press, New York (1966).

[5] a) Kraus, J.D., Antennas, eq. 2-45, McGraw-Hi11, New York (1950).

b) Radio Astronomy, eq. 6-22, McGraw-Hi11, New York (1966).

[6] Ko, H.C., Radio telescope antenna parameters, IEEE Trans. Mi1. Electron., MIL-8, pp. 225-232 (July-October 1964).

[7] Tai, C.T., and Pereira, C.S., An approximate formula for calculating the directivity of an antenna, IEEE Trans. Ant. Propagat., AP-24, pp. 235-236 (March 1976). 
NBS-114A (REV. 2-8G)

U.S. DEPT. OF COMM.

BIBLIOGRAPHIC DATA

SHEET (See instructions)

1. PUBLICATION OR
REPORT NO.
NBSIR $81-1644$

2. Performing Organ. Report No

3. Publication Date

4. TITLE AND SUBTITLE

An Approximate Expression for the Principal Beamwidths of Directive Antennas in Terms of Aperture Fields

5. $\operatorname{AUTHOR}(S)$

Arthur D. Yaghjian

6. PERFORMING ORGANIZATION (If joint or other than NBS, see instructions)

7. Contract/Grant No.

NATIONAL BUREAU OF STANDARDS

DEPARTMENT OF COMMERCE

8. Type of Report \& Period Covered

WASHINGTON, D.C. 20234

9. SPONSORING ORGANIZATION NAME AND COMPLETE ADDRESS (Street, City. Stote, ZIP)

10. SUPPLEMENTARY NOTES

Document describes a computer program; SF-185, FIPS Software Summary, is attached.

11. ABSTRACT (A 200-word or less factual summary of most significant information. If document includes a significant bibliography or literature survey. mention it here)

An approximate, but general, formula for the half-power principal beamwidths of directive antennas is derived from the Fourier transform of the aperture electric field. The derivation, which can be accomplished by expanding the transform in a Taylor series, is greatly streamlined by introducing a Gaussian beam approximation. The formula which also relates beamwidth to directivity indirectly through simple integrations of the aperture field, reduces to a very compact expression in terms of the zero and second order moments of the aperture distribution for linearly polarized uniformly phased aperture fields.

12. KEY WORDS (Six to twelve entries; alphabetical order; capitalize only proper names; and separate key words by semicolons) Antenna beamwidths; aperture fields; directive antennas.

13. AVAILABILITY

X. Unlimited

For Official Distribution. Do Not Release to NTIS

Order From Superintendent of Documents, U.S. Government Printing Office, Washington, D.C. 20402.

X Order From National Technical Information Service (NTIS), Springfield, VA. 2216I
14. NO. OF

PRINTED PAGES

14

15. Price

$\$ 5.00$ 

